\title{
Cierre Percutáneo de Orejuela izquierda con dispositivo LAmbre
}

\author{
Rodrigo Muñoz ${ }^{1,2}$, Ignacio Cruz González ${ }^{3}$, Marcelo Gómez Silva ${ }^{4}$, Javier Rodriguez Collado ${ }^{3}$, Jean Nuñez ${ }^{3}$. \\ 1 Unidad de Hemodinámica, Hospital Sótero del Río, Santiago, Chile. \\ 2 Clínica Alemana Santiago, Chile. \\ 3 Departamento de Cardiología, Complejo Asistencial Universitario de Salamanca, Instituto de Investigación Biomédica \\ de Salamanca (IBSAI), España. \\ 4 Unidad de Hemodinámica, Hospital Dr. Gustavo Fricke, Viña del Mar, Chile.
}

\section{Percutaneous closure of the left atrial appendage using the LAmbre device}

Atrial fibrillation $(\mathrm{AF})$ is an increasing health care problem associated with thromboembolic risk about $5 \%$ per year, with high mortality and morbidity when associated to stroke. Oral anticoagulants (OAC) are the treatment of choice for preventing ischemic stroke in patients with nonvalvular atrial fibrillation (NVAF). However, these drugs are associated with an increased risk of serious complications such an intracranial hemorrhage (ICH). In this context percutaneous closure of the left atrial appendage (LAA) is an effective therapeutic alternative to OACs, with an increasing success rate. Novel devices might allow or facilitate the procedure in some anatomically and technically complicated cases. Two patients with a complex morphology of the LAA, in which the LAmbre (Lifetech Scientific [Shenzhen] Co. Ltd.) device was implanted with good technical and clinical results are presented

Key words: left atrial appendage occlusion, percutaneous, LAmbre device. 
La Fibrilación auricular (FA) es la arritmia cardíaca más prevalente. La FA no valvular (FANV) está asociada con un riesgo significativo de embolia y accidente cerebrovascular (alrededor de 5\% por año) correspondiendo a la segunda etiología de accidente cerebrovascular e incrementando la morbilidad y mortalidad, especialmente en la población de edad avanzada. Los anticoagulantes orales (ACO) son los fármacos de elección para la prevención del accidente vascular isquémico secundario a FANV ${ }^{1}$. No obstante, estos fármacos presentan un riesgo de complicaciones serias como hemorragia intracraneal ${ }^{2}$. En este contexto, el cierre percutáneo de la orejuela izquierda (OI) es una alternativa terapéutica a la anticoagulación oral para la prevención de accidentes cerebrovasculares isquémicos en pacientes con FANV ${ }^{3,4}$. Actualmente los 2 dispositivos más usados para el cierre percutáneo de OI son el Watchman (Boston Scientifict) y Amulet (St Jude Medical), con tasas de éxito del procedimiento cada vez mayores y con progresiva disminución de las complicaciones ${ }^{5,6}$. Sin embargo, existen casos complejos en donde dispositivos con nuevos diseños podrían facilitar el implante.

A continuación presentamos nuestra experiencia en dos casos en que usamos el dispositivo LAmbre (Li- fetech Scientific [Shenzhen] Co. Ltd.), que se usa con creciente frecuencia en Asia y Europa.

\section{Caso Clínico 1:}

Mujer de 84 años con antecedentes de hipertensión arterial (HTA), dislipidemia (DLP), Diabetes Mellitus tipo 2 (DM2), además de FA y valvulopatía aórtica con estenosis severa. El ecocardiograma transtorácico evidenció ventrículo izquierdo de tamaño y función normal (FEVI 65\%), hipertrofia concéntrica leve con septum de $12 \mathrm{~mm}$. La aurícula izquierda estaba severamente dilatada y la válvula mitral con anillo calcificado sin estenosis, con insuficiencia leve a moderada. La válvula aórtica era trivalva fibrocalcificada, con insuficiencia leve y estenosis severa (gradiente máximo $95 \mathrm{~mm} \mathrm{Hg}$, medio $50 \mathrm{~mm} \mathrm{Hg}$ ). El riesgo embólico por CHADS-VASc 5 , era $7.2 \%$ por año Se inició anticoagulación con antagonistas de vitamina K. La paciente presentó púrpura y se cambió el tratamiento a rivaroxaban, (NOAC) pero presentó episodios de hemorragia digestiva. Ingresó con hematocrito de $27 \%$ y hemoglobina de $9 \mathrm{gr} / \mathrm{dl}$. El score HAS-BLEED era 3 implicando un riesgo de sangrado $>3.74 \%$ por año.

Se decidió efectuar el cierre percutáneo de OI. En primera instancia se usó dispositivo Amulet (St Jude

Fig. 1: A) ETE $130^{\circ}$ OI Bilobulada; B) Angiografa Ol; C) Flecha indica Amulet protruyendo de Ol; D) Angiografia de Amulet protruyendo de OI; E) Despliegue bien posicionado de LAmbre; F) ETE a $0^{\circ}$ LAmbre correctamente posicionado ocluyendo OI.
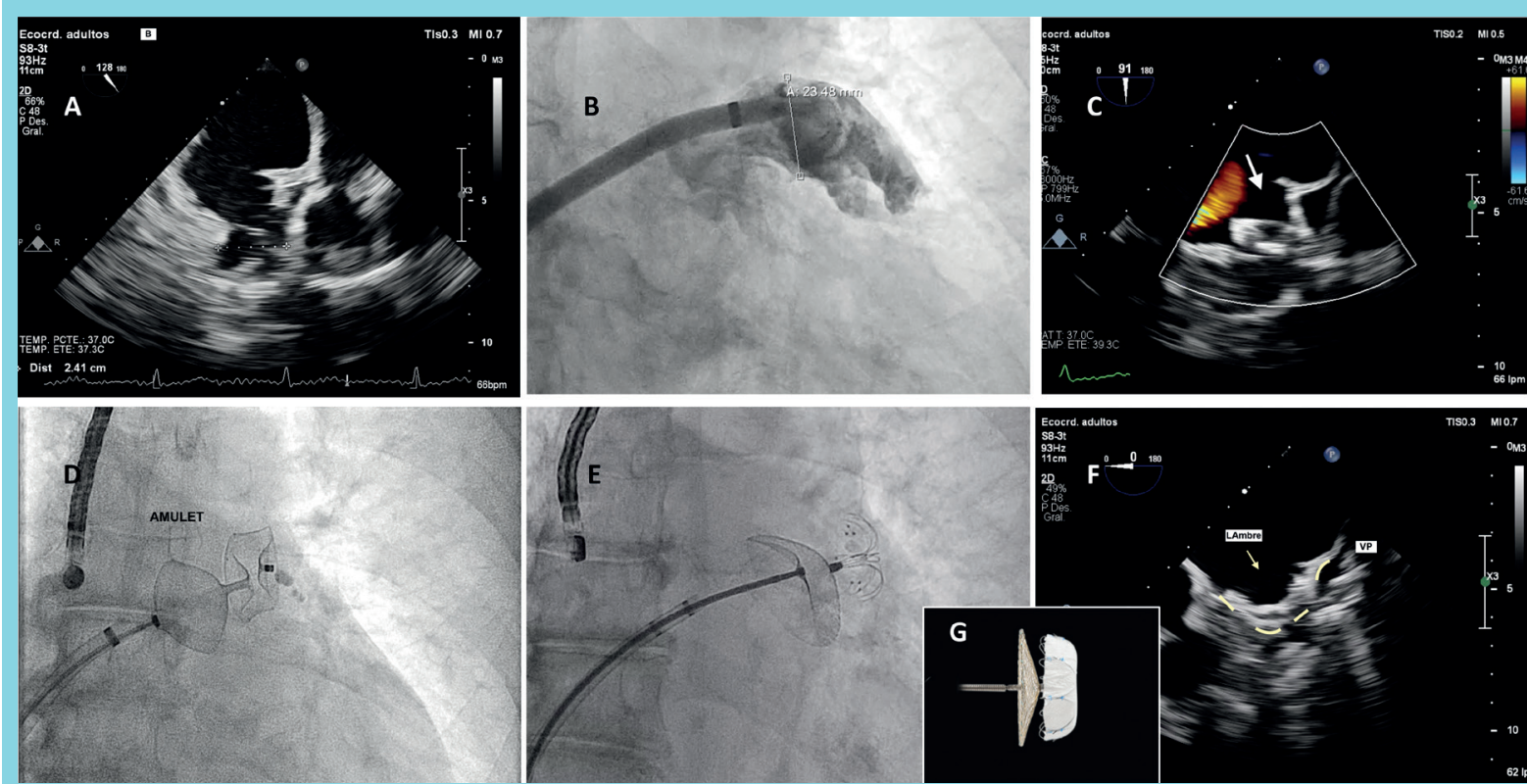
Fig. 2: A), B) ETE y C) Muestra Angiografía de OI que confirman anatomia tipo chicken wing; D) Despliegue parcial de dispositivo LAmbre; E) Inyección de contrante muestra cierre completo de Ol; F) ETE confirma cierre completo de OI.
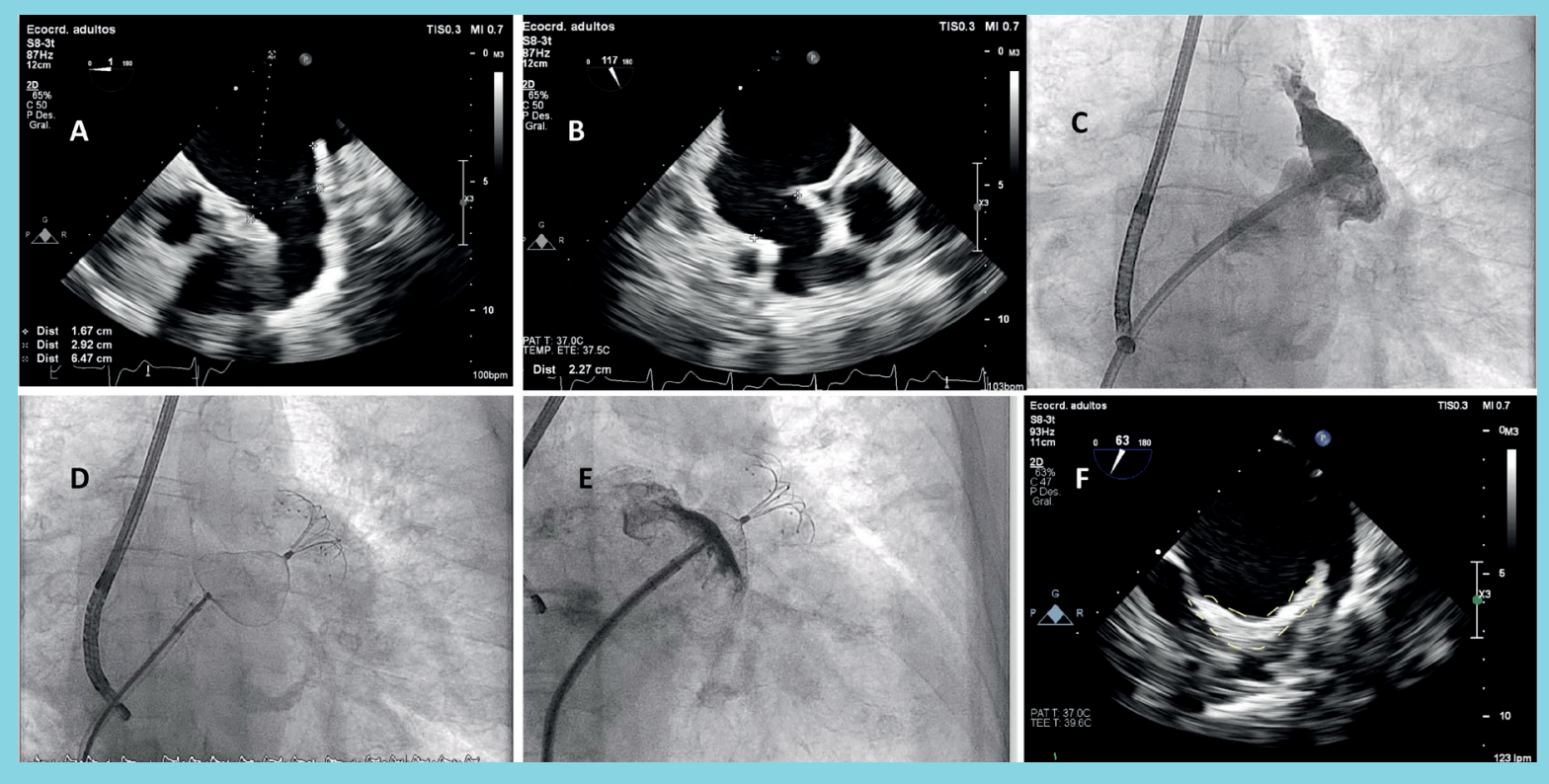

Medical) bajo sedo analgesia, guiado por sonda de ecografía transesofágica micro Philips. Mediante punción venosa femoral derecha guiada por ultrasonido se efectuó la punción trans septal con vaina de St. Jude Swartz SL0 y aguja de Brockenbrough sin complicaciones. Se avanzó guía 0,035” de alto soporte Amplatzer, posicionándola en vena pulmonar superior izquierda. Luego, se intercambió por vaina de liberación de dispositivo $\mathrm{N}^{\mathrm{o}} 14 \mathrm{~F}$, Asistida con técnica de pigtail se posicionó vaina de liberación en OI. Con guía ecográfica y angiográfica se intentó liberar dispositivos Amulet $\mathrm{N}^{\circ} 25$ y luego 28 sin éxito. Ello se atribuyó a la presencia de una OI bilobulada, al ancho insuficiente de los lóbulos y la poca profundidad de la parte proximal. Al intentar desplegar los dispositivos estos prolapsaban ("escupian") hacía afuera, explicable por la necesidad de una profundidad de al menos $12.5 \mathrm{~mm}$. Se hizo un nuevo intento, esta vez con dispositivo LAmbre (Lifetech Scientific [Shenzhen] Co. Ltd.). 24/36 mm. Posicionamos la vaina frente de ostium de OI y desplegamos el paraguas parcialmente, empujando lentamente el dispositivo desde la vaina. Luego se empujó todo el sistema "en bloque" hacía la zona de aterrizaje para permitir floración del paraguas y agarre de los ganchos de retención a las paredes de la OI. Retiramos la vaina para exponer el disco y cubrimos el ostium de la OI, empujando el cable de entrega hacia delante. Comprobamos la colocación del dispositivo mediante angiografía y ecografía y se realizó prueba con un tirón suave, aplicando tensión al cable de entrega para garantizar estabilidad del dispositivo. El encaje logrado fue adecuado, con cobertura total del ostium de la OI. La paciente evolucionó sin complicaciones. (Figura 1).

\section{Caso Clínico 2:}

Mujer de 82 años, portadora de obesidad (IMC: 31 $\mathrm{kg} / \mathrm{m} 2$ ) y FA permanente, en tratamiento con NOAC (Apixaban). Presentó dos episodios de hemorragia digestiva alta en un mes, por lo que se suspendió anticoagulación y se propuso el cierre de OI. Tenía un CHADS - VASc de 4 (4\% de riesgo embólico anual) y HAS-BLED Score 3 (riesgo de sangrado 3.74\%/año).

Se demostró una anatomía de OI tipo "chicken wing" con ostium grande. Se efectuó el cierre de orejuela con técnica similar a la descrita en el Caso número 1. Se utilizó un dispositivo de cierre LAmbre (Lifetech Scientific [Shenzhen] Co. Ltd.) tamaño 34/38 mm. No hubo complicaciones (Figura 2).

En un control con ecocardiografía trans esofágica 3D a los 45 días post implante se demostró, en ambos casos, que el dispositivo se acopló satisfactoriamente a la anatomía de la OI, sin trombos en la superficie, ni leak residual (Figura 
3 A). En ambos casos, dado los antecedentes de sangrado, se mantuvo tratamiento con aspirina $100 \mathrm{mg} /$ día.

\section{Discusión:}

En pacientes con FA y alto riesgo embólico y contraindicación de tratamiento anticoagulante, el cierre de OI es una alternativa factible y segura. Sin embargo, pacientes con OI cuyas anatomías son complejas o presentan ostium grandes, presentan un desafío técnico para el implante de los dispositivos de cierre más ampliamente utilizados, no pudiendo usarse en el cierre de OI con ostium mayores a $31 \mathrm{~mm}$, para Watchman, y "landing zone" mayor a $31 \mathrm{~mm}$ para Amulet. Ambos dispositivos requieren sobredimensionamiento para su estabilización. En estos casos con anatomía de OI bilobulada, o "chicken wing" con ostium grande, se puede lograr el cierre de OI con dispositivo LAmbre, cuyo diseño facilitó el implante. Este es un dispositivo auto expandible a base de nitinol que comprende un paraguas con gancho incorporado y una cubierta conectada con una cintura central corta ${ }^{7}$. La cintura actúa como una conexión articulada y compatible entre la cubierta y el paraguas, lo que permite que la cubierta se adapte a la pared cardíaca. Dentro de sus principales características podemos destacar que se puede usar una vaina de liberación de 8 y 10F, con doble curva distal 45 x $30^{\circ}$ (frente a 12 y $14 \mathrm{~F}$ para el caso de los dispositivos Amulet y Wacthman) con ángulo distal de las vainas $45 \times 45^{\circ}$ ); la disponibilidad de distintos tamaños de la cubierta para el mismo tamaño de paraguas, distancia entre cubierta y paraguas de $4 \mathrm{~mm}$, pero con la cara apendicular de forma convexa y un doble sistema de estabilización (gancho y anclas en forma de U para que se atrapen en las trabéculas). Así, es posible abordar anatomías complejas y tamaños grandes de OI. Además, si es necesario el dispositivo puede recuperarse completamente y volver a desplegarse.

En términos generales la técnica de implantación es similar a la de los otros dispositivos de cierre de OI, exceptuando algunos detalles propios relacionados al diseño del dispositivo.

Creemos que este dispositivo es una alternativa a los disponibles en el mercado. Se destaca el bajo calibre de las vainas lo que puede disminuir las complicaciones vascula-

Fig. 3: A) Ecocardiográfia transesofágico 3D y doppler color a los 45 días post implante caso clínico 1. B) Esquema de implante en diferentes anatomias.
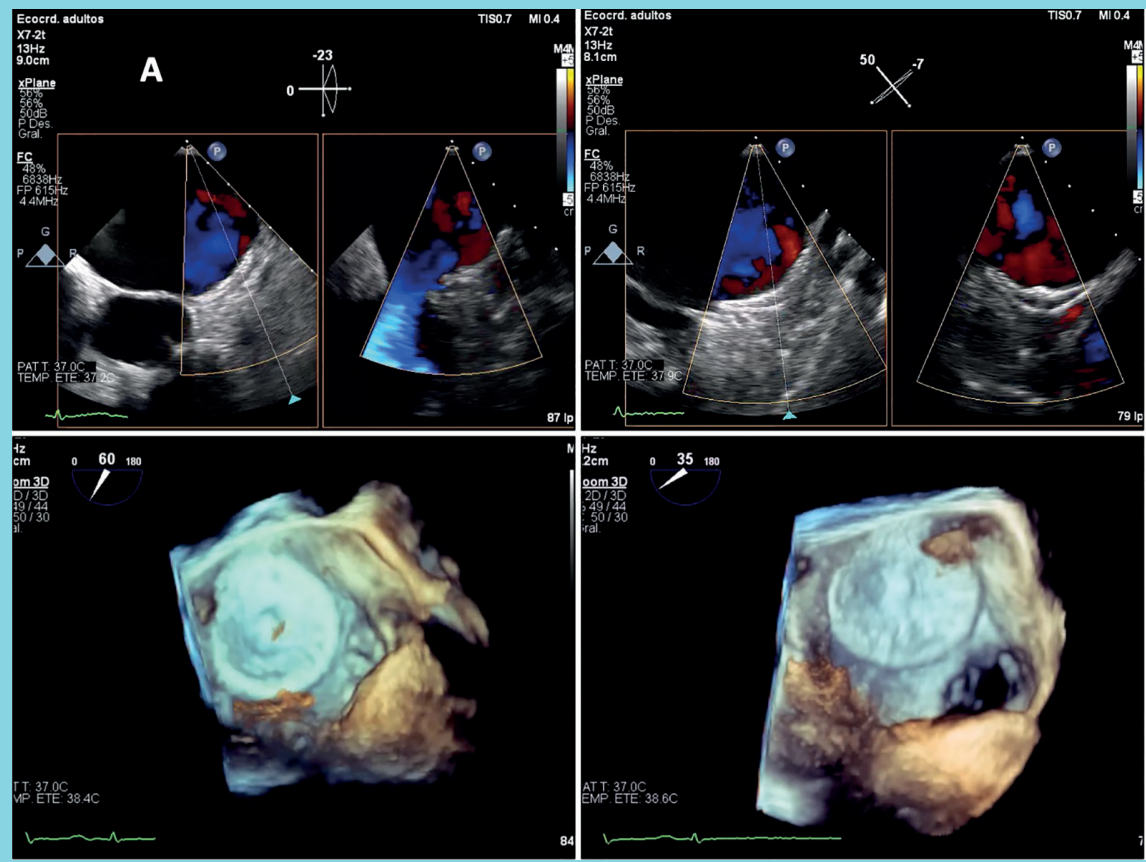

B
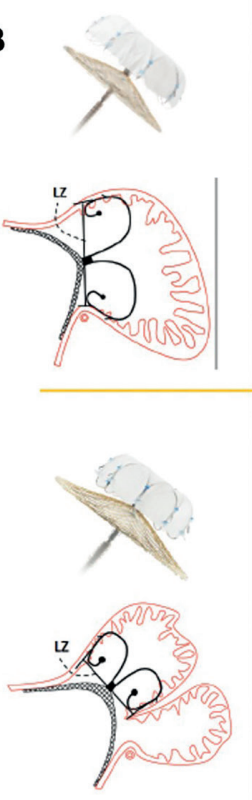
res, la doble curva de su vaina puede disminuir el riesgo de doblez, cuando se intenta alcanzar los lóbulos más anteriores, incluso con punciones trans septales medias (la zona ideal de punción para este dispositivo es ínfero posterior). El doble sistema de estabilización potencialmente disminuiría el número de embolizaciones y facilita el implante al disminuir el número de recapturas. Desde nuestro punto de vista, la característica más importante es la amplia alternativa para distintas anatomías de OI, con grandes cubiertas para paraguas pequeños (ej., paragua/cubierta 22/34 o 26/38 mm), que facilitaría el implante en anatomías de "chicken wing" con zona de implante muy corta o las orejuelas cónicas con gran diferencia de tamaño entre ostium y parte media de la orejuela (Figura 3B).

\section{Referencias}

1. JANUARY CT, WANN LS, ALPERT JS, CALKINS H, CIGARROA JE, CLEVELAND JC, et al. 2014 AHA/ACC/HRS guideline for the management of patients with atrial fibrillation: executive summary: a report of the American College of Cardiology/ American Heart Association Task Force on practice guidelines and the Heart Rhythm Society. Circulation. 2014;130:2071-104.

2. GRYSIEWICZ R, GORELICK PB. Incidence, mortality, and risk factors for oral anticoagulant associated intracranial hemorrhage in patients with atrial fibrillation. J Stroke Cerebrovasc Dis. 2014;23:2479-88.

3. TZIKAS A, GAFOOR S, MEERKIN D, et al. Left atrial appendage occlusion with the Amplatzer Amulet device: an expert consensus step-by-step approach. EuroIntervention. 2016;11:1512.

4. CRUZ-GONZÁLEZ I, RAMA-MERCHAN JC, MARTÍNEZ-PERALTA S, LÓPEZ-MESONERO L, RODRÍGUEZ-COLLADO J, SÁNCHEZ PL. Eficacia y seguridad del cierre percutáneo de orejuela izquierda en pacientes con hemorragia intracraneal. Rev Esp Cardiol. 2017;70:58-60.

5. BOERSMA LV, SCHMIDT B, BETTS TR, et al. Implant success and safety of left atrial appendage closure with the WATCHMAN device: peri-procedural outcomes from the EWOLUTION registry. Eur Heart J. 2016;37:2465-2474.

6. CRUZ-GONZÁLEZ I, ARZAMENDI D, RAMA-MERCHAN JC, PIÑA-GONZÁLEZ P, SÁNCHEZ PL, SERRA A. Left atrial appendage occlusion with the new AmuletTM device: feasibility, safety and short-term efficacy. Rev Esp Cardiol. 2015;68:724-726.

7. LAM YY. A new left atrial appendage occluder (Lifetech LAmbre Device) for stroke prevention in atrial fibrillation. Cardiovasc Revasc Med 2013;14: 134-6.

8. LAM YY, YAN BP, DOSHI SK, et al. Preclinical evaluation of a new left atrial appendage occluder (Lifetech LAmbreTM device) in a canine model. Int J Cardiol. 2013;168:3996-4001. 